INPLASY

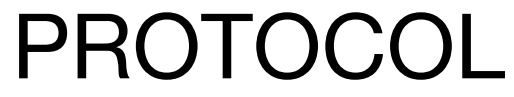

To cite: Tang et al.

Acupuncture therapy on chronic fatigue syndrome based on radar plot: $\mathrm{A}$ protocol for an overview of systematic reviews. Inplasy protocol 202060052. doi: 10.37766/inplasy2020.6.0052

Received: 14 June 2020

Published: 14 June 2020

Corresponding author: Lijie Tang

tang_tcm@163.com

Author Affiliation:

Chengdu University of

Traditional Chinese Medicine

Support: None.

Review Stage at time of this submission: Preliminary searches.

Conflicts of interest:

The authors have no conflicts of interest to disclose.

\section{Acupuncture therapy on chronic fatigue syndrome based on radar plot: A protocol for an overview of systematic reviews}

Tang, LJ1; Jiang, T2; Zhu, FY3; Liu, ZK; Tang, Y5; Wu, X6.

Review question / Objective: To overview the reporting and methodology quality of systematic reviews/meta-analysis on acupuncture in the treatment of chronic fatigue syndrome (CFS).

Condition being studied: Chronic Fatigue Syndrome (CFS) is a heterogeneous disease characterized as disabling fatigue, sleep disorders and cognitive disorders. The peak age of onset is between $\mathbf{2 0}$ and $\mathbf{4 5}$ years old, which seriously affects life and work.It is a major public health problem with high cost. Most of the systematic evaluation/meta-analysis studies have shown that acupuncture, as a complementary replacement therapy for CFS, is safe, effective, simple, and without side effects. The protocol aims to summarize the report quality and methodological quality of systematic evalution/meta-analysis of CFS in acupuncture treatment, and to realize the visual evaluation with the aid of radar chart.

INPLASY registration number: This protocol was registered with the International Platform of Registered Systematic Review and Meta-Analysis Protocols (INPLASY) on 14 June 2020 and was last updated on 14 June 2020 (registration number INPLASY202060052).

\section{INTRODUCTION}

Review question / Objective: To overview the reporting and methodology quality of systematic reviews/meta-analysis on acupuncture in the treatment of chronic fatigue syndrome (CFS).
Condition being studied: Chronic Fatigue Syndrome (CFS) is a heterogeneous disease characterized as disabling fatigue, sleep disorders and cognitive disorders. The peak age of onset is between 20 and 45 years old, which seriously affects life and work.It is a major public health problem with high cost. Most of the systematic evaluation/meta-analysis 
studies have shown that acupuncture, as a complementary replacement therapy for CFS, is safe, effective, simple, and without side effects. The protocol aims to summarize the report quality and methodological quality of systematic evalution/meta-analysis of CFS in acupuncture treatment, and to realize the visual evaluation with the aid of radar chart.

\section{METHODS}

Search strategy: (Acupunctural OR acupuncture OR needle OR 'acupuncture therapy' OR 'acupuncture and moxibustion' OR Zhenjiu OR Zhenci OR Dianzhen OR scalp-acupuncture OR 'auricular acupuncture' OR 'catgut embedding' OR 'auricular therapy' OR acupressure OR acupoint) AND ('chronic fatigue syndrome' OR fatigue OR 'fatigue syndrome, chronic' OR 'Myalgic Encephalomyelitis') AND ('Meta analys' OR 'Systematic review').

Participant or population: Patients with chronic fatigue syndrome.

Intervention: Acupuncture, electroacupuncture, fire needle, auricular acupuncture, catgut embedding, auricular therapy, acupressure, acupoint injection or any combination of the above.

Comparator: Placebo, sham acupuncture, drugs, psychotherapy or other conventional treatments.

Study designs to be included: All systematic reviews and meta-analysis of randomized controlled trials.

Eligibility criteria: Published systematic reviews which were reported in Chinese or English, and meet the "PICOS", will be considered for inclusion in this overview.

Information sources: A comprehensive search of 8 electronic databases, including the Cochrane Library, EMBASE (OVID), Web of Science, PubMed, Wanfang Database, China National Knowledge Infrastructure (CNKI), Chinese Scientific Journals Database (VIP), and Chinese Biomedical
Database (CBM) from inception to 1 June 2020 will be conducted without language restriction.

Main outcome(s): Total effective rate, fatigue scale like Chalder's Fatigue Scale (CFQ), Fatigue Scale-14 (FS-14).

Additional outcome(s): 36-Item Short-Form Health Survey (SF-36), Hamilton Anxiety Scale(HAMA), Hamilton Depression Scale(HAMD), Visual Analogue Scale (VAS).

Data management: Excel2019 will be used to create two tables, one of which includes extraction of data according to the title, first author, publication year, number of included MAs/SRs, sample size, intervention measures, control interventions, outcomes, results, GRADE, AMSTAR, and PRISMA. Another table will be used to multivariate analysis of radar map which extraction of data contains first author, publication year, type of study, AMSTAR, PRISMA, homogeneity, publication bias and rank average.

Quality assessment / Risk of bias analysis: The quality of evidence will be evaluated by using the GRADE, A Measurement Tool to Assess Systematic Reviews (AMSTAR) will be used to evaluate the methodological quality, Preferred Reporting Items for Systematic Reviews and Meta-Analysis (PRISMA) will be used to assess reporting quality, and Adobe Illustrator $C C$ will be used to draw and optimize radar plot.

Strategy of data synthesis: Will provide a narrative description of the findings of the included systematic reviews (SRs). Tables will be produced to detail the included studies and their outcomes. In addition, we will synthesis these reviews and provide pooled treatment effects for all SRs which include the following outcomes: Total effective rate, fatigue scale like Chalder's Fatigue Scale (CFQ), Fatigue Scale-14 (FS-14) , 36-Item Short-Form Health Survey (SF-36), Hamilton Anxiety Scale(HAMA), Hamilton Depression Scale(HAMD), and Visual Analogue Scale (VAS). For each of our outcomes we will perform a sub-group analysis comparing acupuncture VS drugs, 
acupuncture combined with other VS drugs. If necessary, the results will combine in a meta-analysis, the statistical analyses were conducted using the RevMan5.3 software. The summary effect size was estimated by using mean difference (MD) with $95 \%$ confidence intervals (Cl) for continuous outcomes.

Subgroup analysis: If feasible, subgroup analysis will be performed according to gender, different types of acupuncture methods, severity of condition, or intervention period, etc.

Sensibility analysis: No sensitivity analysis required in overview.

Language: Without language restriction.

Country(ies) involved: China.

Keywords: Acupuncture; Chronic Fatigue Syndrome; Overview; AMSTAR; PRISMA;

Radar plot; Multiple evaluation. 